\title{
Why inflammatory phenotyping is necessary for successful drug evaluation in asthma and COPD
}

\author{
Peter G. Gibson ${ }^{1,2}$
}

Affiliations: ${ }^{1}$ Centre for Asthma and Respiratory Diseases, University of Newcastle, Newcastle, and ${ }^{2}$ Dept of Respiratory and Sleep Medicine, Hunter Medical Research Institute, John Hunter Hospital, Newcastle, Australia.

Correspondence: P.G. Gibson, Dept of Respiratory and Sleep Medicine, Hunter Medical Research Institute, John Hunter Hospital, Locked bag \#1, Hunter Mail Centre, Newcastle, NSW, 2310, Australia. E-mail: peter.Gibsonanewcastle.edu.au

@ERSpublications

Pathway specific drug trials must account for prevalence of the pathway by subject selection or by adjusting sample size http://ow.ly/nx4k9

Identifying and treating the eosinophilic subtype of asthma has now achieved renewed importance for clinicians and scientists involved in clinical trials. This has happened following the near-demise of an antiinterleukin (IL)-5 monoclonal antibody (mepolizumab) as a candidate for asthma treatment [1] and its resurrection in trials based around using induced sputum eosinophils to identify a treatment responsive phenotype $[2,3]$. The improvement in outcome was spectacular. An effect size close to zero was converted to a massive effect size of a 50\% reduction in asthma exacerbations, simply by using assessment of clinical status and eosinophils to identify a treatment responsive phenotype. The spotlight is now firmly on clinical trial design in airway diseases and how to identify disease subtypes for testing new therapies. An article by DASGUPTA et al. [4], in this issue of the European Respiratory Journal, addresses this issue by reporting a systematic review of the use of one biomarker, sputum eosinophils, in randomised controlled trials over the past 10 years, and assesses the implications for study design, analysis and sample size in particular.

For a long time, asthma pharmacotherapy has been based around $\beta_{2}$-agonists and corticosteroids, which are regularly reformulated to tweak their clinical efficacy. The promise of new blockbuster treatments has remained both a hope and a necessity, but elusive. Basic scientists have elucidated pathobiological pathways in asthma and, in collaboration with pharmaceutical chemists and immunobiologists, pathway specific treatments have been developed. Once a drug passes the safety hurdle, the next step has been for clinical trials to demonstrate drug efficacy in people with asthma, a phase 3 trial. While every step in this chain is critical, by the time a drug is ready for phase 3 trials, the intellectual and financial investment has been massive and an error at this stage is particularly costly. It is against this background that we need to view modern drug development in asthma and chronic obstructive pulmonary disease (COPD).

What we have learnt from the mepolizumab story is that drugs designed to target a very specific pathway actually target a very specific pathway. If that pathway is not important in the people tested, then the drug has little or no effect. The logical next step is to ask how these pathways and their importance, i.e. clinical relevance, can be identified. Part of the answer lies in the use of biomarkers. The principle of this approach is to use a biomarker to identify the people in whom the pathobiological pathway is active, termed an endotype [5], and then treat them with the pathway specific therapy. We are seeing a technological revolution in biomarker discovery, with the application of 'omics technologies to this area. A recent example is the use of periostin, a serum biomarker identified using transcriptomics of IL-13 stimulated

Received: March 042013 | Accepted: March 212013

Conflict of interest: Disclosures can be found alongside the online version of this article at www.erj.ersjournals.com 
bronchial epithelial cells [6], and linking this to treatment response to lebrikizumab, a monoclonal antibody that targets the IL-13 pathway [7]. In addition to defining an endotype, i.e. subject selection, the biomarker can also be used as an outcome measure. This is useful as it confirms that the treatment is actually hitting the target, and may permit a smaller sample size while issues of drug dosing and effect size estimation are being established.

In this paradigm, sample size calculations are relevant in two ways: firstly in identifying the endotype, i.e. subject selection, and secondly, in measuring the drug effect. The article by DASGUPTA et al. [4] addresses the second of these issues. Their systematic literature review identified 20 randomised controlled trials in asthma and COPD over the past decade that used sputum eosinophils as an outcome measurement. They show that sample size estimates can be affected by the method of data representation, as well as by study design, the specific statistical method of analysis, the estimate of the minimal clinically important difference (MCID) and its variance. Although induced sputum sampling requires relatively simple technology, it can be a difficult technique for some centres to establish. There are many areas of possible variation in the protocols used for induction, processing and counting. Despite these differences, the induced sputum eosinophil count appears surprisingly robust to these variations. Results can be expressed in several ways, such as post-intervention $\%$ - pre-intervention \%, fold change (post-intervention \%/pre-intervention \%) and percentage change from baseline, and DASGUPTA et al. [4] evaluate these three different methods. Percentage change from baseline appears an unstable measure. Fold change and absolute percentage change both have good statistical measurement properties and are recommended for use.

The issue of what constitutes a MCID requires more work. DASGUPTA et al. [4] propose a MCID of a 15\% absolute change, which is a large change and may not be widely applicable. It performed well in the particular study design they evaluate, where refractory eosinophilic asthma patients undergo corticosteroid withdrawal. But this is a rare group who are undergoing a very specific study protocol of corticosteroid withdrawal in addition to testing the add-on therapy. Their proposed MCID also seems to conflict with other data linking a sputum eosinophil threshold of $>3 \%$ with a clinically important increase in exacerbation risk. While fold change lacks immediate clinical interpretation, it does have a defined MCID that can be applied across a range of absolute percentage counts.

This work also underscores how the prevalence of an endotype within a population will have a major influence on sample size, and unless this is adequately addressed, wrong conclusions may be drawn about drug efficacy. For example, if a drug, say an anti-IL-5 therapy, reduces asthma exacerbations by $50 \%$ in eosinophilic asthma, then the prevalence of eosinophilic asthma in the study population will influence the observed overall effect size and, consequently, the study power and sample size requirements. While the effect size may be $50 \%$ in a population where all participants have eosinophilic asthma, if the prevalence of this endotype is reduced by half, as in many clinical settings, then the overall effect size will be reduced by half, i.e. to $25 \%$, having a major impact on power and sample size estimates.

The recognition of heterogeneity in airway disease has major implications for pharmacotherapy. The most promising news is that by accounting for this issue, drug development can proceed in a more predictable way and rational decisions can be made about new therapies. Pathway specific therapies need to account for the prevalence of that pathway in the population under study, either by subject selection or by adjustment of sample size estimates. Biomarkers are an important part of this endeavour, and the work by DASGUPTA et al. [4] shows the necessary analysis paradigm required to make best use of biomarkers in this area.

\section{References}

Walsh GM. Mepolizumab and eosinophil-mediated disease. Curr Med Chem 2009; 16: 4774-4778.

Nair P, Pizzichini MMM, Kjarsgaard M, et al. Mepolizumab for prednisone-dependent asthma with sputum eosinophilia. N Engl J Med 2009; 360: 985-993.

Pavord ID, Korn S, Howarth P, et al. Mepolizumab for severe eosinophilic asthma (DREAM): a multicentre, double-blind, placebo-controlled trial. Lancet 2012; 380: 651-659.

4 Dasgupta A, Zhang S, Thabane L, et al. Sample sizes for clinical trials using sputum eosinophils as a primary outcome. Eur Respir J 2013; 42: 1003-1011.

5 Anderson GP. Endotyping asthma: new insights into key pathogenic mechanisms in a complex, heterogeneous disease. Lancet 2008; 372: 1107-1119.

6 Woodruff PG, Boushey HA, Dolganov GM, et al. Genome-wide profiling identifies epithelial cell genes associated with asthma and with treatment response to corticosteroids. Proc Natl Acad Sci USA 2007; 104: 15858-15863.

7 Corren J, Lemanske RF, Hanania NA, et al. Lebrikizumab treatment in adults with asthma. N Engl J Med 2011; 365: $1088-1098$. 